\title{
Implementation of a trauma-informed, evidence-informed intervention for Latinx families experiencing interpersonal violence and child maltreatment: protocol for a pilot randomized control trial of SafeCare ${ }^{\oplus}{ }^{\circledR}$
}

\author{
Danielle L. Fettes ${ }^{1,2^{*}}$ (D), Gregory A. Aarons ${ }^{1,2}$, Valerie Brew ${ }^{3}$, Karla Ledesma ${ }^{3}$ and Jane Silovsky ${ }^{4}$
}

\begin{abstract}
Background: A consistently demonstrated overlap exists between the occurrence of domestic violence and child maltreatment, yet these issues are historically addressed by distinct systems and programming. The randomized control trial pilot study presented in this article adapts, implements, and tests a new approach for addressing family violence for Latinx families with co-occurring risk for domestic violence and child maltreatment. In doing so, this pilot study addresses the clear need for collaboration between the two fields and focuses on Latinx families, who often face specific challenges regarding seeking and receiving needed services. The primary aim of the current study is a pilot implementation of SafeCare $+^{\oplus}$, an evidence-based parenting curriculum (SafeCare ${ }^{\oplus}$ ) augmented with a healthy relationships curriculum (SafeCare ${ }^{\circledR}$ ). The objectives are a reduction of family violence, improved communication, and a healthy home environment for children in Latinx families with co-occurring domestic violence and child maltreatment.

Methods: This protocol outlines a feasibility, randomized control trial to examine the potential efficacy of SafeCare+. The pilot study is divided into two phases. Components of phase one involve developing a detailed implementation and evaluation plan, including a community needs assessment, determining screening and outcome measures, and assuring all components are culturally appropriate for the target population. Phase two implements the randomization of parents, who are involved in the child welfare system and referred for in-home parenting services, into SafeCare+ or SafeCare as usual. Participants complete assessments regarding mental health, provider-parent relationship, interpersonal violence experiences, and fidelity to the intervention. Analyses will focus on improvement on target outcomes for the intervention group, as well as comparison to the control group.

(Continued on next page)
\end{abstract}

\footnotetext{
* Correspondence: dfettes@health.ucsd.edu

${ }^{1}$ Department of Psychiatry, University of California, 9500 Gilman Drive, \#0812,

La Jolla, San Diego, CA 92093, USA

${ }^{2}$ Child and Adolescent Services Research Center, San Diego, CA, USA

Full list of author information is available at the end of the article
}

(c) The Author(s). 2020 Open Access This article is licensed under a Creative Commons Attribution 4.0 International License, which permits use, sharing, adaptation, distribution and reproduction in any medium or format, as long as you give appropriate credit to the original author(s) and the source, provide a link to the Creative Commons licence, and indicate if changes were made. The images or other third party material in this article are included in the article's Creative Commons licence, unless indicated otherwise in a credit line to the material. If material is not included in the article's Creative Commons licence and your intended use is not permitted by statutory regulation or exceeds the permitted use, you will need to obtain permission directly from the copyright holder. To view a copy of this licence, visit http://creativecommons.org/licenses/by/4.0/ The Creative Commons Public Domain Dedication waiver (http://creativecommons.org/publicdomain/zero/1.0/) applies to the data made available in this article, unless otherwise stated in a credit line to the data. 
(Continued from previous page)

Discussion: This study will provide evidence on the feasibility and potential effectiveness of an early intervention program aimed at improving communication skills and mental health and reducing incidents of violence for Latinx parents who are involved with the child welfare service system. The findings of the study will inform the decision to progress to a full scale, definitive randomized control trial to test the effectiveness of an intervention, delivered as part of home visitation, for improving outcomes for families with histories of domestic violence.

Trial registration: ClinicalTrials.gov, NCT03041558; registered 2 February, 2017—retrospectively registered.

Keywords: Domestic violence, Child welfare, Feasibility studies, Pilot projects, Early intervention, Home visitation

\section{Background}

Child maltreatment and domestic violence (DV) are two vital public health concerns, often with considerable overlap and in extensive need of amelioration. Over 4 million cases of suspected child maltreatment were reported to state child protective service systems in the USA in 2017, with approximately 674,000 confirmed cases of maltreatment. The most common form of child maltreatment in the USA is neglect, accounting for over $74 \%$ of substantiated cases [1]. The individual and societal impacts of child maltreatment are well documented, as child trauma is associated with negative social, emotional, behavioral, and health effects on children and youth [2] at a notable economic cost [3-5]. The impacts of DV are similarly alarming. In the USA, over 1 in 3 (or 42.6 million) women experienced violence by an intimate partner over the course of a lifetime, with 6.6 million women reporting violence within the last year [6]. Approximately 15.5 million children are exposed to DV each year, with over $50 \%$ of DV incidents occurring in households with children [7].

\section{Co-occurrence of domestic violence and child maltreatment}

Two decades of research have clearly documented a strong overlap between DV and child maltreatment, with national research indicating a 30 to $60 \%$ co-occurrence rate $[8,9]$. These forms of family violence have a strong likelihood of co-occurring because they share several common risk factors, such as young maternal age, low level of education, and low socioeconomic status, among others $[10,11]$. Children who have witnessed or experienced violence in their homes face an elevated risk of cognitive and emotional problems [12]. They often experience negative outcomes such as poor school performance, grade retention, juvenile delinquency, and teenage pregnancy [8]. Further, children who reside in homes with DV may show warning signs of stress, fear, and trauma [13].

While child maltreatment clearly has an adverse effect on healthy child development, the presence of DV can also interfere with parenting, placing children at additional risk. Children living in households with DV are more likely to experience emotional, physical, and sexual abuse [14]. Perpetrators often attempt to control their adult partners through methods such as intimidation, undermining their parental authority, or using children against the other parent [15]. Children may be injured when they attempt to intervene in a DV incident or get struck by objects intended to injure or frighten the parental victim. And, in the absence of physical abuse, DV may deteriorate the family environment and increase stress, impacting parents' capacity to attend to their children's needs.

Taken together, there is a clear need for collaborative efforts to provide appropriate services and to identify families struggling with relationship dynamics earlier in the cycle of family violence. Despite the growing awareness of the co-occurrence of DV and child maltreatment, however, programs and systems have historically responded separately. Existing parenting programs designed to address child maltreatment rarely include training in managing DV-related issues. However, the presence of DV has been shown to affect domains of improvement that are targeted by parenting programs. For example, a common element of many parenting programs is a focus on improving parent-child interactions. DV perpetrators often damage the parent-child relationship by victim-blaming and breeding distrust in what is typically the mother's ability to protect her children [16]. Including content that addresses DV in the context of parenting programs creates a unique opportunity to both deliver services that acknowledge the complexity of the intersection of DV and parenting and provide early intervention to children and parents at risk for long term, negative outcomes.

\section{Need for culturally relevant and trauma-informed services for Latinx families}

In addition to addressing service needs for families with co-occurring risk for DV and child maltreatment, the current study specifically focuses on Latinx families. The characteristics or consequences of DV among different cultures may vary. Attention to cultural differences among DV survivors can attune services to particular psychological, emotional, spiritual, economic, legal, and 
social needs. Cultural factors unique to the Latinx community that may impact the manifestation of DV include concepts such as machismo or marianismo [17]. These gender ideals encourage men to be dominant in intimate relationships and for women to demonstrate submissive and passive behaviors $[18,19]$. Some evidence demonstrates that the effects of DV on Latina women lead to significantly greater trauma-related symptoms and depression as compared to non-Latina women [20].

The Latinx community in the USA covers a wide range of experiences, from recent immigrants with limited command of the English language, to families with generations of citizenship. Latina immigrants may have difficulty recognizing or responding to DV due to patriarchal values and beliefs in familismo, or family loyalty [21]. Even when DV is identified as an issue, Latina survivors can often face other obstacles in seeking help. Common barriers to help-seeking behaviors include lack of English proficiency, fear of deportation, and perception that law enforcement will not respond to domestic disputes [22]. Latinx families may face a range of additional risk factors such as recent immigration, resulting in great distance from traditional support systems, challenges related to cultural expectations, religion, and previous experiences of violence [23]. These factors may elevate traumatic stress reactions, indicating a clear need for collaborative efforts that focus on (1) identifying these families earlier in the cycle of family violence, (2) supporting them to garner the necessary skills to keep themselves and their families safe and healthy, and (3) providing safety planning and violence prevention.

\section{In-home parenting programs as a unique platform to provide early intervention for DV}

The primary aim of the current study is a pilot implementation and testing of culturally relevant, traumainformed programming to lead to a reduction of family violence, a resulting increase in family stability, improved communication, and a healthy home environment for children in Latinx families with co-occurring DV and child maltreatment. Specific objectives include the following:

1. Increased access to culturally specific, traumainformed, evidence-informed interventions.

2. Increased understanding of the needs of the target population and how to meet them.

3. Improved collaboration, policies, and practices for effectively meeting the needs of the target population.

4. Reduced incidents of DV at case closure.

5. Improved mental health at case closure.

6. Improved communication skills at case closure.
The intervention being implemented is SafeCare+ $(\mathrm{SC}+)$, an evidence-based (EB) training curriculum for parents referred for child maltreatment, enhanced with the healthy relationships (HR) module. SafeCare (SC) has been shown to be widely accepted among the Latinx community [24]. The HR enhancement is a promising practice that may prevent and/or reduce family violence among Latinx families. The study will test the efficacy of the SC+ model, contributing to the DV field's evidence base of effective practices that improve outcomes for Latinx families impacted by both DV and child maltreatment. The evaluation of the pilot implementation will impact and inform advocacy and practice change.

\section{Methods}

\section{Setting}

This randomized-control trial (RCT), pilot study was embedded within a large-scale, National Institute of Mental Health-funded study, the Interagency Collaborative Teams to Scale-Up Evidence Based Practice (ICT) [25]. The ICT study was a system-wide, mixed-methods implementation study focused on understanding inner and outer context factors associated with the effective implementation and sustainment of SC delivered to child welfare-involved parents, in a large, diverse child welfare service (CWS) system. The ICT study components (which include an assessment of the feasibility and acceptability of $\mathrm{SC} / \mathrm{SC}+$, the impact of service attrition on study outcomes, and fidelity to the intervention) serve as a foundation for the current pilot RCT.

The scale up of SC occurred across multiple communitybased organizations (CBOs). These $\mathrm{CBOs}$ are contracted by CWS to deliver parent training, such as $\mathrm{SC}$, via an in-home service delivery platform. Families involved with CWS are referred to the $\mathrm{CBO}$ s to receive SC because they had children between the ages of 0 and 11 and child neglect was the primary referral reason. As an EB curriculum, SC is structurally and behaviorally prescriptive on how its curriculum is to be delivered to the targeted parents by the home visitors. Most often, parents participate in the SC curriculum individually with the home visitor. Parent participant eligibility criteria for the ICT study were (1) at least 18 years of age; (2) were referred for child neglect; and (3) had at least one child in the family under age 12 . Over $90 \%$ of eligible parents enrolled in the ICT study.

\section{Design}

The pilot study is divided into two phases. During phase one, the first year of the study, community and academic partners collaborate to develop a detailed evaluation plan. The evaluation centers on the effective implementation of $\mathrm{SC}+$ and measurement of relevant outcomes during phase two of the project-the RCT, pilot study. Components of phase one include (1) a community 
needs assessment, (2) refining the target population, (3) designing the evaluation plan, (4) determining screening and outcome measures, (5) conducting focus groups with key stakeholders, including DV providers and Latinx survivors, to assure that the chosen interventions and screening and assessment tools are culturally appropriate for the target population, and (6) ensuring the proper training of direct service staff. For phase two, the RCT pilot study will enroll at least 160 parents either into $\mathrm{SC}$ or $\mathrm{SC}+$, allocating a minimum of 80 parents per condition. Parents will be randomized to $\mathrm{SC}$ or $\mathrm{SC}+$ and within home visitor, such that every other eligible parent will be assigned to $\mathrm{SC}$ or $\mathrm{SC}+$ within home visitor. The nested structure will be accounted for in multi-level analyses, see Fig. 1 (SafeCare+ study flow diagram).

\section{Eligibility criteria}

Participants will be included in the RCT pilot study if the following criteria are met: (1) currently receiving SC services, meeting ICT inclusion criteria; (2) a primary caregiver identifying as Latinx; (3) over 18 years of age; and (4) at risk for DV. The criterion for risk of DV was intentionally set to be broadly inclusive of families who are struggling with psychological violence. Clients will be excluded from participation if there are immediate safety risks in the family.

\section{Ethical considerations}

Participants providing data in the study are service clients receiving family preservation/family reunification services (i.e., $\mathrm{SC}$ via home visitation) through partnering CBOs in the CWS system. These services are most often targeted at concerns over child neglect, and services are designed to strengthen the capacity of families to care for young children. Parental participation in the research study is voluntary, and parental data is maintained separate from CWS records. Parental declination to participate in the research study has no impact on service receipt-clients will receive CWS services independent of participation in the study.

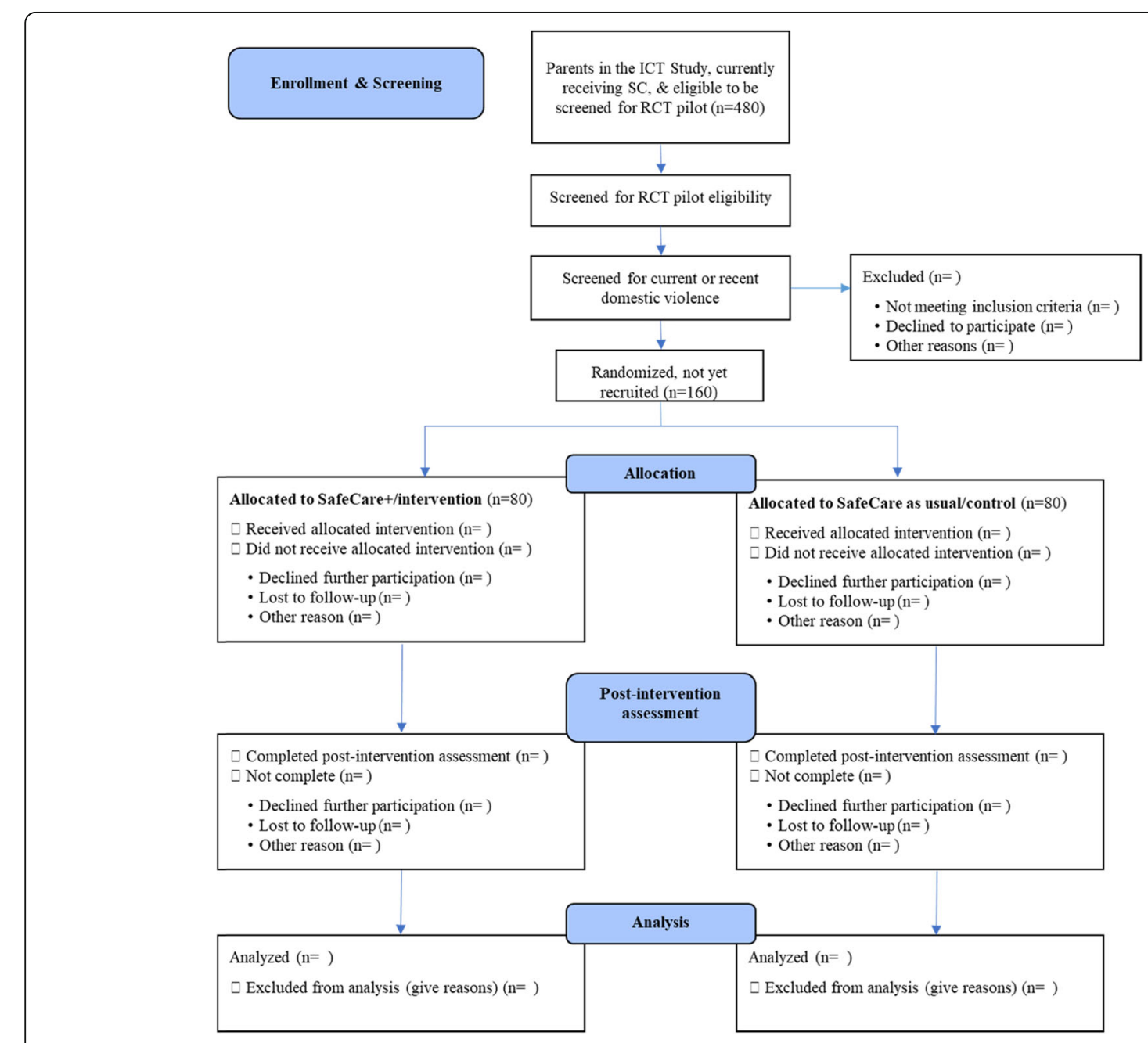

Fig. 1 SafeCare+ study flow diagram 


\section{Interventions}

\section{Control group: SafeCare}

SafeCare (SC) is an EB parenting curriculum, delivered via home visitation, for parents with preschool or school-age children who are at risk of or have been reported for child neglect, physical abuse, or both. SC targets improvement in skills related to home safety, health, and parent-child interaction [26]. These modules include a baseline assessment, training in knowledge and skills, and follow-up assessments to monitor change. Delivery of the program is based on the principles of behavioral analysis-service providers are trained to model skills, engage in ongoing measurement of observable behaviors, and to give constructive feedback during skills practice. SC program completion typically takes 18 to 20 home visiting sessions over 6 months. A number of studies attest to SC effectiveness and provide evidence on improved parental outcomes, including better management of their child's health, increased home safety, and more positive and sensitive parent-child interaction [2628 ]. In addition, $\mathrm{SC}$ has been demonstrated to be efficacious for reducing CWS recidivism relative to usual care [29]. Participants randomized to the control group will receive the $\mathrm{EB}, \mathrm{SC}$ as usual.

\section{Experimental group: SafeCare with Healthy Relationships (SafeCare+)}

Participants randomized to the experimental group will receive $\mathrm{SC}+$, an enhanced version of the $\mathrm{SC}$ program, which includes the healthy relationships (HR) curriculum, specifically designed for early intervention with family violence. The HR curriculum is intended to help parents recognize abusive patterns, develop healthier relationships, and improve communication skills with a range of adults, including intimate partners, family members, friends, and with their children. The HR module (1) provides information about how relationships can affect health and well-being for parent and child; (2) reviews qualities of healthy and unhealthy relationships; (3) assesses the type and quality of the parent's important relationships; (4) empowers the parent to be an active participant in improving the quality of their personal relationships; and (5) teaches communication, problem-solving, and interpersonal skills to facilitate enhancing the quality of their personal relationships. The HR module is delivered in a similar skills-based format as other SC components, with ongoing measurement and feedback in areas of communication, problem solving, and interpersonal interactions.

\section{Feasibility outcomes}

Acceptability of the intervention will be examined in both phases of the study. First, focus groups with the target population participants will determine the perceived fit of the intervention for the population. Focus groups will also provide information regarding the variety of experiences, barriers, and strengths that exist within various sub-groups of the target population (e.g., younger and older parents, recent immigrants). This information will be used to design and test various approaches to service delivery to meet the varied needs of the target population and to determine the feasibility for expanding the target population to include a prevention base for families at risk of DV. Second, satisfaction will be established by program participants on all intervention components. In addition to acceptability, SC+ limited effectiveness will be ascertained by comparisons between the experimental and control groups on key outcome measures, set to achieve at minimally medium effect size. Finally, practicality (i.e., perceived sustainability within the existing infrastructure) will be explored at the conclusion of the study via interviews with providers and service system leadership.

\section{Measures}

All measures are client-reported and will be collected via a secure, web-based data entry system, on an internetenabled tablet that service providers bring to each visit. At the end of each SC or SC+ visit, providers activate the participant survey by logging into the secure webbased data entry system on their assigned tablet computer and selecting the appropriate client identifier (a number randomly generated by the secure system). Once the survey has been activated, the service provider will give the client the tablet computer to complete the measures privately. In the infrequent circumstance that two parents are simultaneously participating in services, the assessments are completed by each, separately, while the other parent is engaged with the home visitor. All measures are available in both English and Spanish.

\section{Screening measures}

Hurt, Insult, Threaten, Scream (HITS) [30] is a 4-item instrument for DV screening. Items ask respondents how often their partner physically hurt, insulted, threatened with harm, and screamed at them. A score of 10.5 (range 4 to 20) was demonstrated to reliably differentiate victims of domestic abuse from family practice patients. The current pilot study includes parents who report a score of 7 or higher.

\section{Outcome measures}

$\mathrm{SC}+$ model fidelity is assessed via client report, with clients completing a fidelity questionnaire at the end of every SC or SC+ session. The fidelity survey was adapted from the National SafeCare Training and Research Center training checklists, and the fidelity measure has been validated over the course of SC implementation [31]. 
Overall, monitoring of fidelity allows for measuring the effectiveness of the intervention as designed, as well as any practice changes which are naturally occurring and may need to be addressed.

The Conflict Tactics Scales (CTS2)-short form [32, 33] measures both the extent to which partners in a dating, cohabiting, or marital relationship engage in psychological and physical attacks on each other, as well as use of reasoning or negotiation to deal with conflicts. Participants are asked about the frequency of concrete acts and events within the past 6 months for their baseline CTS2 assessment, with the time frame changed to assessing if these acts and events occur within the past 2 months for the post-intervention assessment.

The Center for Epidemiological Studies Depression Scale (CES-D) [34] is a 20-item questionnaire, which assesses depressive symptomology. Designed as a short self-report tool, each item asks about the last week and is responded to using a 4-point format. The CES-D has high internal consistency and adequate test-retest repeatability.

Relationship self-efficacy (RSE) [35] is a 35-item measure evaluating relationship maintenance self-efficacy beliefs. Thirteen items relevant to the content of HR are included, across the three domains of emotional control, differentiation, and mutuality. Emotional control denotes the ability to appropriately regulate negative feelings with others. Differentiation is the capacity to assert clear interpersonal boundaries and express the need for separateness. Mutuality is defined as being able to provide care and receive support.
Working Alliance Inventory (WAI) [36] assesses working alliance between parents and their service providers. The current study employs the short-form, 12-item version of the WAI [37] and includes the components of goal, task, and bond. Goal is the extent to which the client and provider agree on goals of services. Task is the degree to which the client and provider agree on ways of achieving stated goals. Bond denotes mutual liking between client and provider.

Relationship status is a brief instrument created by the study team and intended to assess the respondent's current intimate partner relationship or rationale for not currently engaging in one.

Healthy relationships satisfaction is a 14-item assessment of perspectives about the prescribed HR content, delivery of services, and service provider.

Figure 2 illustrates the framework of the research approach.

\section{Data collection points for outcome measures}

Data collection for $\mathrm{SC} / \mathrm{SC}+$ fidelity will be conducted at each session of the intervention, throughout the course of service delivery. The CES-D is assessed at intake into $\mathrm{SC}$, at the end of the intervention, and at the end of the study. The CTS-2, WAI, RSE, and relationship status measures are assessed at the beginning and conclusion of receiving the intervention (i.e., the HR curriculum, or the comparison time frame for the control condition). HR satisfaction is assessed at the conclusion of receiving the intervention, see Fig. 3 (SPIRIT figure for SafeCare+ trial for data collection timings).

Program: ACYF Healthy Relationships

Goal: To contribute to the DV fields evidence base of effective practices that improve outcomes for Latinx Families impacted by both DV and child abuse, by testing the efficacy of the SafeCare+ model for this population.

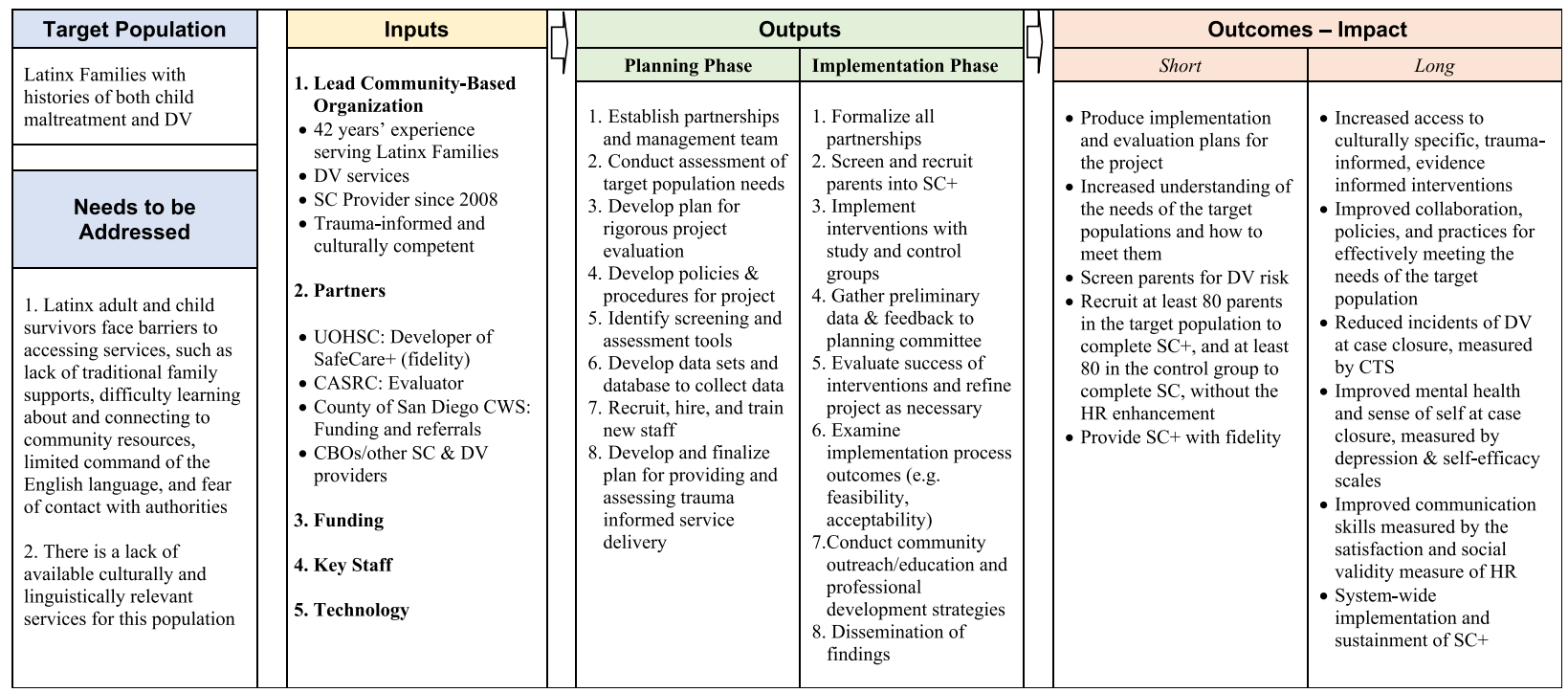

Fig. 2 Logic model for the SafeCare+ pilot study 


\begin{tabular}{|c|c|c|c|c|c|c|}
\hline & \multicolumn{6}{|c|}{ Study Period } \\
\hline & $\begin{array}{c}\text { Enrollment } \\
\text { into SafeCare }\end{array}$ & Screening & $\begin{array}{c}\text { Pre- } \\
\text { Intervention } \\
\text { Assessment }\end{array}$ & $\begin{array}{c}\text { Start of } \\
\text { Intervention }\end{array}$ & $\begin{array}{c}\text { Post- } \\
\text { Intervention } \\
\text { Assessment }\end{array}$ & $\begin{array}{l}\text { End of } \\
\text { Study }\end{array}$ \\
\hline \multicolumn{7}{|l|}{ Timepoint } \\
\hline \multicolumn{7}{|l|}{ Enrollment } \\
\hline Eligibility Screen & & $\mathrm{X}$ & & & & \\
\hline Informed Consent & & & $\mathrm{x}$ & & & \\
\hline Demographics & $\mathrm{x}$ & & & & & \\
\hline Random Allocation & & & $\mathrm{X}$ & & & \\
\hline \multicolumn{7}{|l|}{ Interventions } \\
\hline \multicolumn{7}{|l|}{$\begin{array}{l}\text { Experimental: } \\
\text { SafeCare+/Healthy } \\
\text { Relationships }\end{array}$} \\
\hline \multicolumn{7}{|c|}{ Control: Treatment as usual } \\
\hline \multicolumn{7}{|l|}{ Assessments: } \\
\hline CESD & $\mathrm{x}$ & & & $\mathrm{X}$ & $\mathrm{X}$ & $\mathrm{x}$ \\
\hline HITS & & $\mathrm{X}$ & & & & \\
\hline \multicolumn{7}{|l|}{ Fidelity } \\
\hline CTS-2 & & & & $\mathrm{x}$ & $\mathrm{X}$ & \\
\hline WAI & & & & $\mathrm{X}$ & $\mathrm{X}$ & \\
\hline RSE & & & & $\mathrm{X}$ & $\mathrm{x}$ & \\
\hline Relationship Status & & & & $\mathrm{X}$ & $\mathrm{X}$ & \\
\hline HR Satisfaction & & & & & $\mathrm{X}$ & \\
\hline
\end{tabular}

Fig. 3 SPIRIT figure for SafeCare+ trial for data collection timings

\section{Data analyses}

\section{Quantitative analyses}

Primary analyses will be based on generalized linear mixed models [38-40]. Mixed models are necessary for analyzing data from the proposed project because the data will have a three-level, hierarchical data structure in which measurements over time are nested within clients and clients are nested within provider. The models tested involve both fixed and time-varying covariates. All models will be multivariate in nature (i.e., testing all predictors simultaneously). Significance tests will focus on individual regression coefficients from the models. In addition, the final data set will offer rich opportunities for auxiliary or secondary analyses, including exploring the role of fidelity for mediating client outcomes. Dependent variables will be changed in (1) reports of DV on the CTS, (2) depression, (3) relationship selfefficacy, and (4) indicators of fidelity to SC and SC+.

\section{Sample size}

A conservative estimate for the duration of the proposed study is 160 parents, which provides an adequate sample size required to discern improvement, with a medium Cohen's $d$ effect size, on the proposed measures.

\section{Discussion}

This pilot study targets families with co-occurring DV and child maltreatment and will demonstrate the impacts of an early intervention approach for Latinx parents with histories of DV. The study addresses the needs of this target population by providing SC, an EB home visiting program designed for families reported for, or at risk of, child maltreatment, supplemented through the addition of an HR curriculum that has been modified specifically for Latinx families $(\mathrm{SC}+)$. This study design is appropriate for the target population because it provides a culturally and linguistically refined approach that promotes the development of individual skills such as problem solving, active decision-making, conflict resolution, and healthy communication-both for enhanced parenting and within personal relationships. The HR module is intended to help parents learn about the characteristics of healthy and unhealthy relationships so they may begin to examine and improve the quality of their own relationships. It is anticipated that as parents become active agents in creating and maintaining healthy personal relationships, they will provide a safer and more emotionally healthy environment for their children and will enhance their parenting effectiveness. Thus, the 
goals are to improve family stability by reducing conflict, improve communication in all areas of the parents' life, and provide positive modeling for children. All services will promote integrity and self-sufficiency, improve access to resources, and increase the safety of adult and child survivors of DV and child maltreatment.

A primary aim of the RCT, pilot study is to demonstrate the effectiveness of the HR curriculum. A significant advantage of the design of the pilot study is the embedded nature of the RCT into a larger study. Substantial infrastructure support with regard to recruitment and data collection serve as an important foundation to success. A primary challenge of the RCT pilot study is that the inclusion of the HR curriculum into the existing service delivery system extends the length of services by $30 \%$ ( $\sim 2$ months). As a result, parents assigned to the intervention condition may not actually receive the entirety, or in some cases any, of the intervention due to the service requiring more time than the CWS timeline for length of service delivery. A study limitation is that parents with literacy challenges may have difficulty completing the outcome measures to determine effectiveness. In addition, the cultural appropriateness of outcome measures is a primary consideration. The first phase of the study is intended specifically to collaborate with technical assistance providers, community partners, and Latinx parents to ensure that the data collection efforts are aligned with the trauma-informed approach of the study.

Results from this study will inform the ways in which the CWS system of care may be strengthened to sustain long-term safety and well-being of children, while also providing efficacy data for a large, system-wide randomized control trial of the intervention. In addition, service providers are gaining multiple experiences with the provision of trauma-informed practice and intentional approaches to working with children and families with histories of DV. This pilot study provides an important foundational step to determining how DV services may be integrated into CWS home visitation programs, setting a platform for continued work with this population.

\section{Abbreviations}

CBO: Community-based organization; CWS: Child welfare services; DV: Domestic violence; HR: Healthy relationships; RCT: Randomized control trial; SC: SafeCare; SC+: SafeCare with Healthy Relationships

\section{Acknowledgements}

We are grateful for the collaborative relationship with the San Diego County Child Welfare Service System, which provides the training and fiscal support for the implementation of the pilot intervention.

\section{Authors' contributions}

DF: Led the design and execution of this pilot study and wrote the article. GA: Co-led the design and execution of the larger, NIH study, and consulted on the design and execution of the pilot study. VB: Supervised the implementation of the intervention, as well as supported the writing of the article. $\mathrm{KL}$ : Led the implementation of the intervention, as well as supported the writing of the article. JS: Created the healthy relationships curriculum and consulted on the study design. The author(s) read and approved the final manuscript.

\section{Funding}

This article presents independent research funded by the Administration for Children and Families (HHS-2013-ACF-ACYF-EV-0635). Additional funding for the implementation was received by the San Diego Department of Health and Human Services, Child Welfare Services. The views expressed are those of the author(s) and not necessarily those of the ACF or the San Diego HHSA, CWS.

\section{Availability of data and materials}

The datasets generated during and/or analyzed during the current study are not publicly available due to ongoing recruitment and the sensitive nature of the study population, but are available from the corresponding author on reasonable request.

\section{Ethics approval and consent to participate}

The Institutional Review Board of the University of California, San Diego (UCSD), approved the study protocol (IRB\# 110035).

\section{Competing interests}

The authors declare that they have no competing interests.

\section{Author details}

'Department of Psychiatry, University of California, 9500 Gilman Drive, \#0812, La Jolla, San Diego, CA 92093, USA. ${ }^{2}$ Child and Adolescent Services Research Center, San Diego, CA, USA. ${ }^{3}$ South Bay Community Services, San Diego, CA, USA. ${ }^{4}$ Health Sciences Center, The University of Oklahoma, Oklahoma City, OK, USA.

Received: 28 June 2019 Accepted: 8 September 2020

Published online: 08 October 2020

\section{References}

1. Child Maltreatment 2017. U.S. Department of Health \& Human Services, Administration on Children YaF, Children's Bureau; 2019.

2. Swenson CC, Brown EJ, Lutzker JR. Personality disorders in childhood and adolescence. In: Freeman IA, editor. Issues of maltreatment and abuse. Hoboken: Wiley; 2007. p. 229-95.

3. Fang X, Brown DS, Florence CS, Mercy JA. The economic burden of child maltreatment in the United States and implications for prevention. Child Abuse Negl. 2012;36(2):156-65.

4. Peterson C, Florence C, Klevens J. The economic burden of child maltreatment in the United States, 2015. Child Abuse Negl. 2018;86:178-83.

5. Wang C-T, Holton J. Total estimated cost of child abuse and neglect in the United States Chicago. Illinois: Prevent Child Abuse America; 2007.

6. Smith SG, Zhang, X., Basile, K.C., Merrick, M.T., Wang, J., Kresnow, M., Chen, J. The National Intimate Partner and Sexual Violence Survey (NISVS): 2015 Data Brief In: Control NCfIPa, editor. 2018.

7. McDonald R, Jouriles EN, Ramisetty-Mikler S, Caetano R, Green CE. Estimating the number of American children living in partner-violent families. J Fam Psychol. 2006;20(1):137-42.

8. Herrenkohl TI, Sousa C, Tajima EA, Herrenkohl RC, Moylan CA. Intersection of child abuse and children's exposure to domestic violence. Trauma Violence Abuse. 2008;9(2):84-99.

9. McGuigan WM, Pratt CC. The predictive impact of domestic violence on three types of child maltreatment. Child Abuse Negl. 2001;25(7):869-83.

10. Cox CE, Kotch JB, Everson MD. A longitudinal study of modifying influences in the relationship between domestic violence and child maltreatment. J Fam Violence. 2003;18(1):5-17.

11. Schechter SK, J. Early childhood, domestic violence, and poverty: helping young children and their families. lowa City: The University of lowa, Work SoS; 2004.

12. Briggs-Gowan MJ, Estabrook R, Henry D, Grasso DG, Burns J, McCarthy KJ, et al. Parsing dimensions of family violence exposure in early childhood: shared and specific contributions to emergent psychopathology and impairment. Child Abuse Negl. 2019;87:100-11.

13. Knitzer J. Promoting resilience: helping young children and parents affected by substance abuse, domestic violence, and depression in the context of 
welfare reform: Columbia University, Mailman School of Public Health, Poverty TNCfCi; 2000.

14. Holt S, Buckley $\mathrm{H}$, Whelan $\mathrm{S}$. The impact of exposure to domestic violence on children and young people: a review of the literature. Child Abuse Negl. 2008;32(8):797-810.

15. Mbilinyi LF, Edleson JL, Hagemeister AK, Beeman SK. What happens to children when their mothers are battered? Results from a four city anonymous telephone survey. J Fam Violence. 2007;22(5):309-17.

16. Överlien C, Hydén M. Children's actions when experiencing domestic violence. Childhood. 2009:16(4):479-96.

17. Perilla JL, Bakeman R, Norris FH. Culture and domestic violence: the ecology of abused Latinas. Violence Vict. 1994;9(4):325-39.

18. Cianelli R, Ferrer L, McElmurry BJ. HIV prevention and low-income Chilean women: machismo, marianismo and HIV misconceptions. Cult Health Sex. 2008;10(3):297-306.

19. Fuchsel CLM, Murphy SB, Dufresne R. Domestic violence, culture, and relationship dynamics among immigrant Mexican women. Affilia. 2012;27(3): 263-74

20. Edelson MG, Hokoda A, Ramos-Lira L. Differences in effects of domestic violence between Latina and non-Latina women. J Fam Violence. 2007; 22(1):1-10.

21. Reina AS, Lohman BJ, Maldonado MM. "He said they'd deport me": factors influencing domestic violence help-seeking practices among Latina immigrants. J Interpers Violence. 2014;29(4):593-615.

22. Bauer HM, Rodriguez MA, Quiroga SS, Flores-Ortiz YG. Barriers to health care for abused Latina and Asian immigrant women. J Health Care Poor Underserved. 2000;11(1):33-44.

23. Rivera J. Domestic violence against Latinas by Latino males: an analysis of race, national origin, and gender differentials. Boston Coll Third World Law J. 1994;14(2):231-57.

24. Finno-Velasquez M, L. Fettes D, A. Aarons G, S. Hurlburt M. Cultural adaptation of an evidence-based home visitation programme: Latino clients' experiences of service delivery during implementation. J Childrens Serv 2014;9(4):280-294.

25. Hurlburt M, Aarons GA, Fettes D, Willging C, Gunderson L, Chaffin MJ. Interagency collaborative team model for capacity building to scale-up evidence-based practice. Child Youth Serv Rev. 2014;39:160-8.

26. Lutzker JR, Bigelow KM, Doctor RM, Kessler ML. Safety, health care, and bonding within an ecobehavioral approach to treating and preventing child abuse and neglect. J Fam Violence. 1998;13(2):163-85.

27. Bigelow KM, Lutzker JR. Using video to teach planned activities to parents reported for child abuse. Child Fam Behav Ther. 1998;20(4):1-14.

28. Gershater-Molko RM, Lutzker JR, Wesch D. Project SafeCare: improving health, safety, and parenting skills in families reported for, and at-risk for child maltreatment. J Fam Violence. 2003;18(6):377-86.

29. Chaffin M, Hecht D, Bard D, Silovsky JF, Beasley WH. A statewide trial of the SafeCare home-based services model with parents in Child Protective Services. Pediatrics. 2012;129(3):509-15.

30. Sherin KM, Sinacore JM, Li XQ, Zitter RE, Shakil A. HITS: a short domestic violence screening tool for use in a family practice setting. Fam Med. 1998; 30(7):508-12.

31. Chaffin M, Hecht D, Aarons G, Fettes D, Hurlburt M, Ledesma K. EBT fidelity trajectories across training cohorts using the interagency collaborative team strategy. Admin Pol Ment Health. 2016;43(2):144-56.

32. Straus MA, Douglas EM. A short form of the revised conflict tactics scales, and typologies for severity and mutuality. Violence Vict. 2004;19(5):507-20.

33. Straus MA, Hamby SL, Boney-McCoy S, Sugarman DB. The revised conflict tactics scales (CTS2) development and preliminary psychometric data. J Fam Issues. 1996;17(3):283-316.

34. Radloff LS. The CES-D scale: a self-report depression scale for research in the general population. Appl Psychol Meas. 1977;1 (3):385-401.

35. Lopez FG, Morúa W, Rice KG. Factor structure, stability, and predictive validity of college students' relationship self-efficacy beliefs. Meas Eval Couns Dev. 2017:40(2):80-96.

36. Horvath AO, Greenberg LS. Development and validation of the working alliance inventory. J Couns Psychol. 1989;36(2):223-33.

37. Tracey TJ, Kokotovic AM. Factor structure of the working alliance inventory. J Consult Clin Psychol. 1989;1 (3):207-10.

38. Hedeker D, Gibbons RD. Longitudinal data analysis. Hoboken: Wiley; 2006.

39. McCulloch CE, Searle SR. Generalized, linear, and mixed models. New York: Wiley; 2001
40. Raudenbush SW. Hierarchical linear models: applications and data analysis methods. Thousand Oaks: Sage Publications, Inc; 2002.

\section{Publisher's Note}

Springer Nature remains neutral with regard to jurisdictional claims in published maps and institutional affiliations.
Ready to submit your research? Choose BMC and benefit from:

- fast, convenient online submission

- thorough peer review by experienced researchers in your field

- rapid publication on acceptance

- support for research data, including large and complex data types

- gold Open Access which fosters wider collaboration and increased citations

- maximum visibility for your research: over $100 \mathrm{M}$ website views per year

At BMC, research is always in progress.

Learn more biomedcentral.com/submissions 